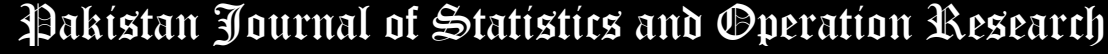

\section{The Log-Balakrishnan-Alpha-Skew-Normal Distribution and Its Applications}

\author{
Sricharan Shah ${ }^{1}$, Subrata Chakraborty ${ }^{1}$, Partha Jyoti Hazarika ${ }^{1 *}$, \\ M. Masoom $\mathrm{Ali}^{2}$
}

\begin{abstract}
* Corresponding Author
1. Department of Statistics, Dibrugarh University, Dibrugarh-786004, Assam, India, charan.shah90@gmail.com, subrata_stats@dibru.ac.in, parthajhazarika@gmail.com

2. Department of Mathematical Sciences, Ball State University, Muncie, IN 47306 USA, mali@bsu.edu
\end{abstract}

\begin{abstract}
In this paper, log-Balakrishnan-alpha-skew-normal distribution is proposed by the methodology of Venegas et al. (2016). Some of its basic distributional properties including the moments also discussed. Also, the appropriateness of this distribution is checked by performing data fitting experiments and comparing with some other known distributions by using Akaike Information Criterion (AIC) and Bayesian Information Criterion (BIC). The Likelihood Ratio test is used for discriminating between log-normal and the proposed distributions.
\end{abstract}

Key Words:Skew Distribution, Alpha-Skew Distribution, Bimodal Distribution, Log-Alpha-Skew Distribution, AIC, BIC.

\section{Introduction}

Log normal distribution is preferred over the normal distribution to model random variable with positive support. Typical uses of log-normal distribution are found in descriptions of fatigue failure, failure rates, and other phenomena involving a large range of data. Another common applications where log-normal distributions are used in finance is in the analysis of stock prices. A random variable $Z$, follows the log-normal distribution $\operatorname{LN}\left(\mu, \sigma^{2}\right)$ with two $\operatorname{parameters}\left(\mu, \sigma^{2}\right)$ if its probability density function (pdf) is defined as $f(z)=\frac{1}{z \sigma} \phi\left(\frac{y-\mu}{\sigma}\right)$, where $y=\log (z), z>$ $0, \mu \in R, \sigma>0$ and $\phi($.$) is the standard normal pdf. The log-normal distributions are positively skewed with long$ right tails due to low mean values and high variances in the random variables.

Vistelius (1960) showed that the chemical element concentrations in soil samples follow asymmetric distribution. Ahrens $(1953,1954 \mathrm{a}, 1954 \mathrm{~b})$ studied the chemical element concentration using many data sets with positive asymmetry. Log-skew-normal distribution with positive support was derived from skew normal was used by MateuFigueras et al. (2004) to deal with geochemical data as the support of the skew-normal distribution in the real line. This distribution is also used by Azzalini et al. (2003), for family income data.

Azzalini (1985) introduced the skew normal distribution with asymmetry parameter $\lambda$ and the pdf given by

$$
f_{Z}(z ; \lambda)=2 \phi(z) \Phi(\lambda z) ; z, \lambda \in R
$$

where $\phi($.$) is define above and \Phi($.$) is cumulative distribution function (cdf) of N(0,1)$.

A useful generalization of the skew normal distribution was proposed as a discussant in Arnold and Beaver (2002) by Balakrishnan (2002) and studied some of its properties. The pdf of the same distribution is

$$
f_{Z}(z ; \lambda, n)=\phi(z)[\Phi(\lambda z)]^{n} / C_{n}(\lambda) ; z, \lambda \in R
$$

where $n$ is a positive integer and $C_{n}(\lambda)=E\left(\Phi^{n}(\lambda U)\right), U \sim N(0,1)$. In case, if $\lambda=1$, this Balakrishnan skew normal distribution reduces to the skew normal distribution of Azzalini (1985). 
Using the idea of Huang and Chen (2007), Elal-Olivero (2010) developed a new form of skew distribution known as alpha skew normal distribution which has both unimodal as well as bimodal behavior and has the following pdf given by

$$
f(z ; \alpha)=\left\{(1-\alpha z)^{2}+1\right\} \phi(z) /\left(2+\alpha^{2}\right) ; z, \alpha \in R
$$

Venegas et al. (2016) studied alpha skew normal distribution and proposed its logarithm form and named it as logalpha skew normal distribution with the pdf

$$
f(z ; \alpha)=\left\{(1-\alpha y)^{2}+1\right\} \phi(y) / z\left(2+\alpha^{2}\right) ; z>0, \alpha \in R, y=\log (z)
$$

Applying the idea of eqn.(2) and eqn.(3), Hazarika et al. (2019) proposed a new form of alpha skew normal distribution known as Balakrishnan alpha skew normal distribution with more flexibility than the distribution in eqn.(3) and exhibits both unimodality and bimodality behaviours. The pdf is given by

$$
f(z ; \alpha)=\frac{\left[(1-\alpha z)^{2}+1\right]^{2}}{C_{2}(\alpha)} \phi(z) ; z, \alpha \in R
$$

where $C_{2}(\alpha)=4+8 \alpha^{2}+3 \alpha^{4}$.

The main aim of this paper includes, first, introducing the log-Balakrishnan alpha skew normal distribution using the idea of Venegas et al. (2016) and discuss its basic properties, second, applying this new proposed distribution which is flexible enough for both unimodality and bimodality behaviors, to real life datasets and third, establish suitability of this proposed distribution over a few other known distributions.

The rest of this paper is organized as follows. In Section 2, we introduce a new form of log-alpha-skew normal distribution and study its mathematical properties. The estimation of parameters and two real life data modeling applications to illustrate the usefulness of the new distribution is presented in Section 3. Finally, concluding remark is noted in Section 4.

\section{The log-Balakrishnan-alpha-skew-normal distribution}

In this section we define a new form of log alpha skew normal distribution and studied some of its distributional properties.

Definition 1: If a random variable $Z$ has a pdf

$$
f(z ; \alpha)=\frac{\left[(1-\alpha y)^{2}+1\right]^{2}}{z C_{2}(\alpha)} \phi(y) ; z>0, \alpha \in R
$$

where $y=\log (z)$ and $C_{2}(\alpha)=4+8 \alpha^{2}+3 \alpha^{4}$, then, it is said to be $\log$-Balakrishnan-alpha-skew-normal distribution with skewness parameter $\alpha$. In the rest of this article we shall refer the distribution in eqn.(6) as $\operatorname{LBASN}_{2}(\alpha)$.

\subsection{Properties of $\operatorname{LBASN}_{2}(\alpha)$ :}

- If $\alpha=0$, then we get the standard log-normal distribution $f(z)=\phi(y) / z$.

- If $\alpha \rightarrow \pm \infty$, then we get the log-bimodal-normal $(L B N(4))$ distribution (see Hazarika et al. 2019) given by $f(z)=\frac{y^{4}}{3 z} \phi(y)$.

- If $Z \sim L B A S N_{2}(\alpha)$, then $-Z \sim \operatorname{LBASN}_{2}(-\alpha)$.

\subsection{Plots of the pdf}

The pdf of $\operatorname{LBASN}_{2}(\alpha)$ distribution for different choices of the parameter $\alpha$ are plotted in Figure 1. It can be seen from Figure 1, that the distribution is positively skewed and higher skewness and kurtosis occur for $0<\alpha<2$. Note that the curves in both plots of Figure 1 look different because of the difference in scaling in vertical axis. 

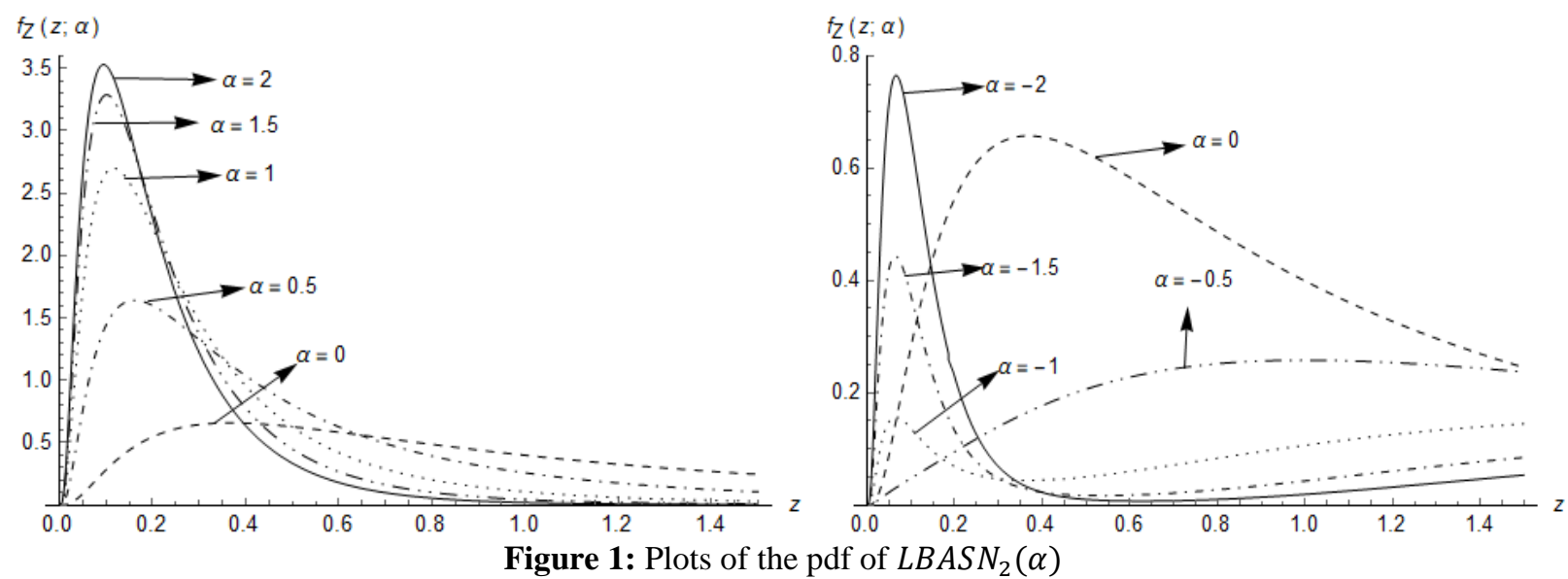

2.3. Mode of $L_{B A S N}(\alpha)$ :

Here, we numerically verify that $\operatorname{LBASN}_{2}(\alpha)$ distribution has at most two modes. First by differentiating the pdf $f(z ; \alpha)$ of $\operatorname{LBASN}_{2}(\alpha)$ distribution with respect to $z$ we get

$$
D f(z ; \alpha)=-\frac{\phi(y)[2+\alpha y(-2+\alpha y)][2+4 \alpha+y(-2(\alpha+1)(2 \alpha-1)+\alpha y(\alpha+\alpha y-2))]}{z^{2} C_{2}(\alpha)} .
$$

Now, the contour of the equation $D f(z ; \alpha)=0$ is drawn and shown in Figure 2 to check that ${ } B A S N_{2}(\alpha)$ distribution has at most two modes or not.
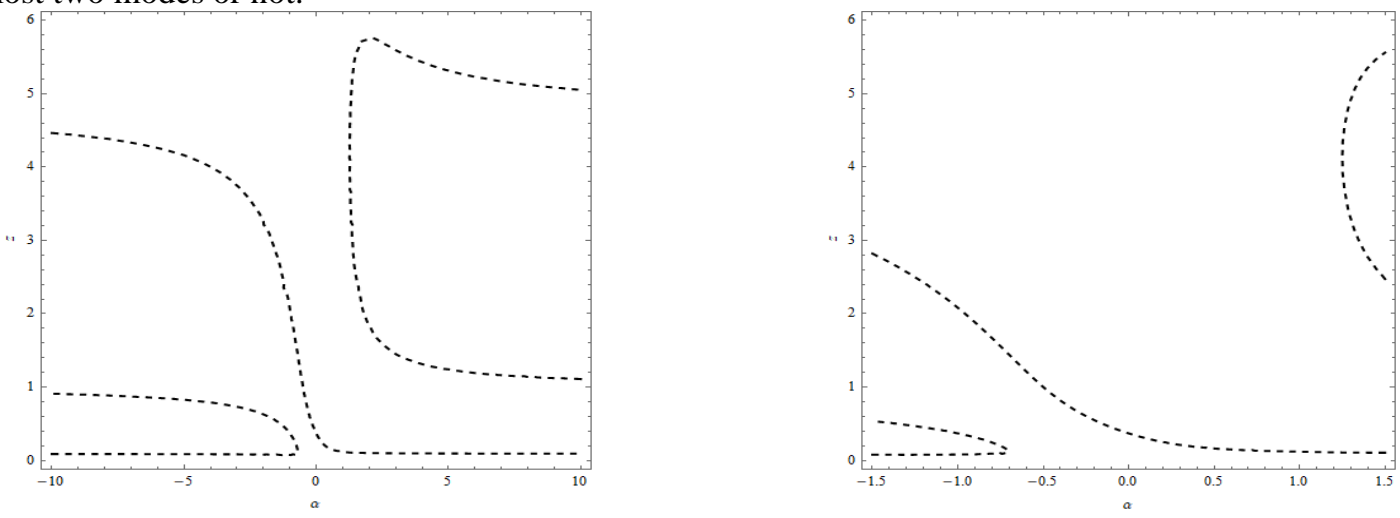

Figure 2: Contour plots of the equation $D f(z ; \alpha)=0$

It can be observed that there is at most three zeros of $D f(z ; \alpha)$ which shows that $\operatorname{LBASN}_{2}(\alpha)$ distribution has at most two modes. Also, for $-0.70<\alpha<1.25, \operatorname{LBASN}_{2}(\alpha)$ remains unimodal.

\subsection{Cumulative distribution function}

Theorem 1: The cdf of $\operatorname{LBASN}_{2}(\alpha)$ distribution is given by

$$
F_{Z}(z)=\frac{\phi(y)\left[e^{y^{2} / 2} 2 C_{2}(\alpha) \sqrt{2 \pi} \Phi(y)-2 \alpha\left\{-8\left(\alpha^{2}+1\right)+\alpha y\left(8+3 \alpha^{2}+\alpha y(-4+\alpha y)\right)\right\}\right]}{2 z C_{2}(\alpha)}, Z>0
$$

Proof: $F_{Z}(z)=P(Z \leq z)$

$$
\begin{gathered}
=\frac{1}{C_{2}(\alpha)} \int_{0}^{z}\left\{\alpha^{4} \log (z)^{4}-4 \alpha^{3} \log (z)^{3}+8 \alpha^{2} \log (z)^{2}-8 \alpha \log (z)+4\right\} \phi(\log (z)) d z \\
=\frac{1}{C_{2}(\alpha)}\left[\alpha^{4}\left[\{3 / 2(1+\operatorname{erf}(\log (z) / \sqrt{2}))\}-\left\{e^{-\frac{\log (z)^{2}}{2}} \log (z)\left(3+\log (z)^{2}\right) / \sqrt{2 \pi}\right\}\right]-4 \alpha^{3}\left[-\left\{e^{-\frac{\log (z)^{2}}{2}}(2\right.\right.\right. \\
\left.\left.\left.+\log (z)^{2}\right)\right\} / \sqrt{2 \pi}\right]+8 \alpha^{2}\left\{1 / 2+1 / 2 \operatorname{erf}(\log (z) / \sqrt{2})-\left(e^{-\frac{\log (z)^{2}}{2}} \log (z) / \sqrt{2 \pi}\right)\right\} \\
\left.-8 \alpha\left\{-\left(e^{-\frac{\log (z)^{2}}{2}} / \sqrt{2 \pi}\right)\right\}+4\{1 / 2(1+\operatorname{erf}(\log (z) / \sqrt{2}))\}\right],
\end{gathered}
$$

where $\operatorname{erf}($.$) is error function.$ 
On simplifying we get the desired results in eqn.(8).

Corollary 1: If we take the limit $\alpha \rightarrow \pm \infty$ of $F_{Z}(z)$ in eqn.(8), then we get the cdf of $L B N(4)$ distribution as $F(z)=$ $\Phi(y)-\frac{y \phi(y)}{z}\left(1+\frac{1}{3} y^{2}\right)$.

The cdf is plotted in Figure 3 for studying variation in its shape with respect to the parameter $\alpha$.

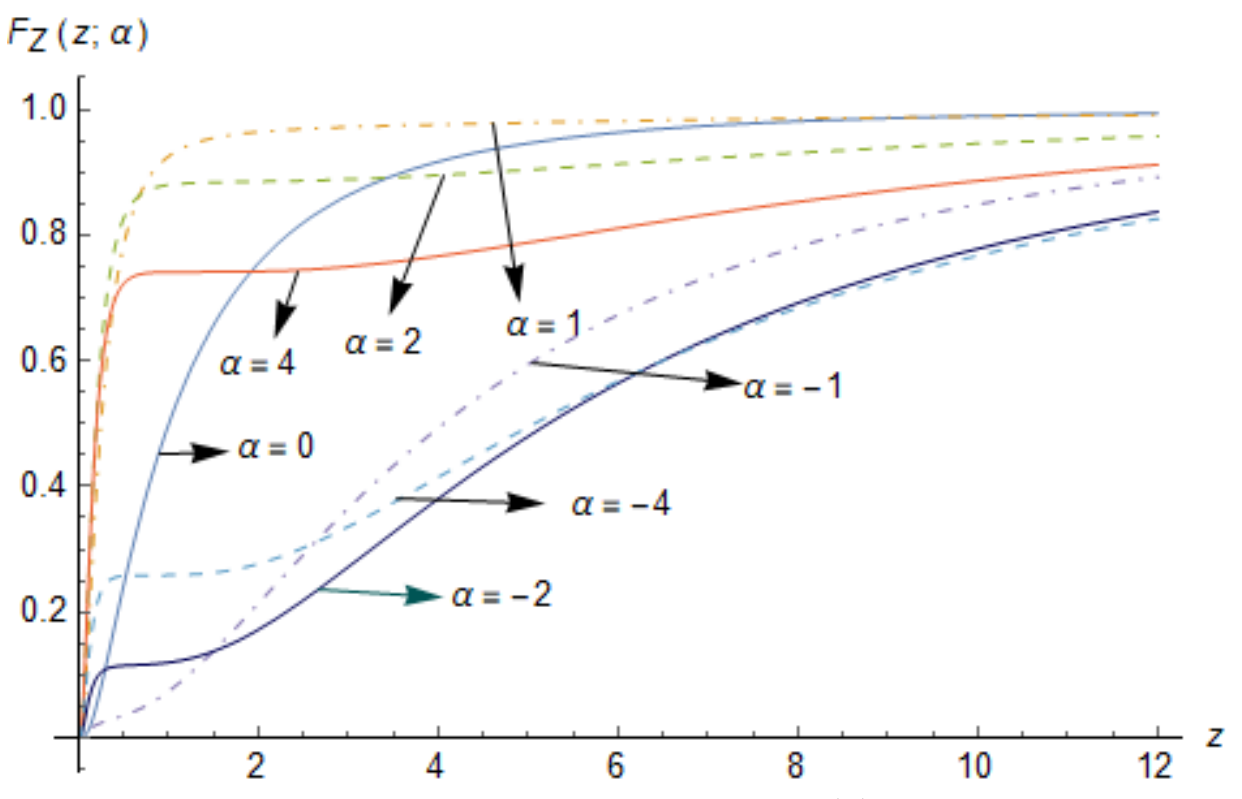

Figure 3: Plots of cdf of $\operatorname{LBASN}_{2}(\alpha)$

\subsection{Moments}

Theorem 2: The $r^{\text {th }}$ order moment of $\operatorname{LBASN}_{2}(\alpha)$ distribution is given by

$$
\begin{gathered}
E\left(Z^{r}\right)=\frac{e^{\frac{r^{2}}{2}}\left(4-8 r \alpha+8\left(1+r^{2}\right) \alpha^{2}-4 r\left(3+r^{2}\right) \alpha^{3}+\left(3+6 r^{2}+r^{4}\right) \alpha^{4}\right)}{C_{2}(\alpha)} . \\
\text { Proof: } E\left(Z^{r}\right)=\frac{1}{C_{2}(\alpha)} \int_{0}^{\infty} z^{r}\left((1-\alpha \log (z))^{2}+1\right)^{2} \phi(\log (z)) d z \\
=\frac{1}{C_{2}(\alpha)} \int_{0}^{\infty} z^{r}\left\{\alpha^{4} \log (z)^{4}-4 \alpha^{3} \log (z)^{3}+8 \alpha^{2} \log (z)^{2}-8 \alpha \log (z)+4\right\} \phi(\log (z)) d z \\
=\frac{1}{C_{2}(\alpha)}\left[\alpha^{4}\left\{e^{r^{2} / 2}\left(3+6 r^{2}+r^{4}\right)\right\}-4 \alpha^{3}\left\{e^{r^{2} / 2} r\left(3+r^{2}\right)\right\}+8 \alpha^{2}\left\{e^{r^{2} / 2}\left(1+r^{2}\right)\right\}-8 \alpha\left\{e^{r^{2} / 2} r\right\}+4\left\{e^{r^{2} / 2}\right\}\right] .
\end{gathered}
$$

On simplifying we get the desired results as in eqn.(9).

From eqn.(9) putting $r=1,2,3$, and 4 , we respectively get

$$
\begin{aligned}
& E(Z)=\frac{2 \sqrt{e}[2+\alpha(-4+\alpha(8+\alpha(-8+5 \alpha)))]}{C_{2}(\alpha)} \\
& E\left(Z^{2}\right)=\frac{e^{2}[4+\alpha(-16+\alpha(40+\alpha(-56+43 \alpha)))]}{C_{2}(\alpha)} \\
& E\left(Z^{3}\right)=\frac{2 e^{9 / 2}\left[2+\alpha\left(-12+\alpha\left(40-72 \alpha+69 \alpha^{2}\right)\right)\right]}{C_{2}(\alpha)}
\end{aligned}
$$

and

$E\left(Z^{4}\right)=\frac{e^{8}[4+\alpha(-32+\alpha(136+\alpha(-304+355 \alpha)))]}{C_{2}(\alpha)}$

Hence,

$V(Z)=\frac{e[-4[2+\alpha(-4+\alpha(-8+5 \alpha)))]^{2}+e C_{2}(\alpha)\{4+\alpha(-16+\alpha(40+\alpha(-56+43 \alpha))\}]}{C_{2}(\alpha)}$. 
Remark 1: The $r^{\text {th }}$ order moment of $\operatorname{LBASN}_{2}(\alpha)$ distribution can also be derived from the moment generating function (mgf) of Balakrishnan alpha skew normal $B A S N_{2}(\alpha)$ distribution of Hazarika et al. (2019) as discussed below.

The pdf and the mgf of the random variable $X \sim B A S N_{2}(\alpha)$ are respectively given by

and

$$
f_{X}(x ; \alpha)=\frac{\left[(1-\alpha x)^{2}+1\right]^{2}}{C_{2}(\alpha)} \phi(x) ; x, \alpha \in R
$$

$$
M_{X}(t)=\frac{e^{\frac{t^{2}}{2}}\left[4-8 t \alpha+8 \alpha^{2}\left(1+t^{2}\right)-4 t \alpha^{3}\left(3+t^{2}\right)+\alpha^{4}\left(3+6 t^{2}+t^{4}\right)\right]}{C_{2}(\alpha)} .
$$

If $Z \sim \operatorname{LBASN}_{2}(\alpha)$ and $X \sim B A S N_{2}(\alpha)$ then it can be verified that

which is same as in eqn.(9).

$$
\begin{gathered}
E\left(Z^{r}\right)=E\left[\exp (r X]=M_{X}(r)\right. \\
=\frac{e^{\frac{r^{2}}{2}}\left[4-8 r \alpha+8 \alpha^{2}\left(1+r^{2}\right)-4 r \alpha^{3}\left(3+r^{2}\right)+\alpha^{4}\left(3+6 r^{2}+r^{4}\right)\right]}{C_{2}(\alpha)} .
\end{gathered}
$$

Remark 2: The bounds for mean and variance can be derived numerically by optimizing $E(Z)$ and $\operatorname{Var}(Z)$ with respect to $\alpha$ as $0.595216 \leq E(Z) \leq 7.36516$ and $1.44567 \leq \operatorname{Var}(Z) \leq 77.9201$. Also, to study their behavior we have plotted the mean and the variance respectively in Figure (4) and Figure (5). These plots also reveal these bounds.

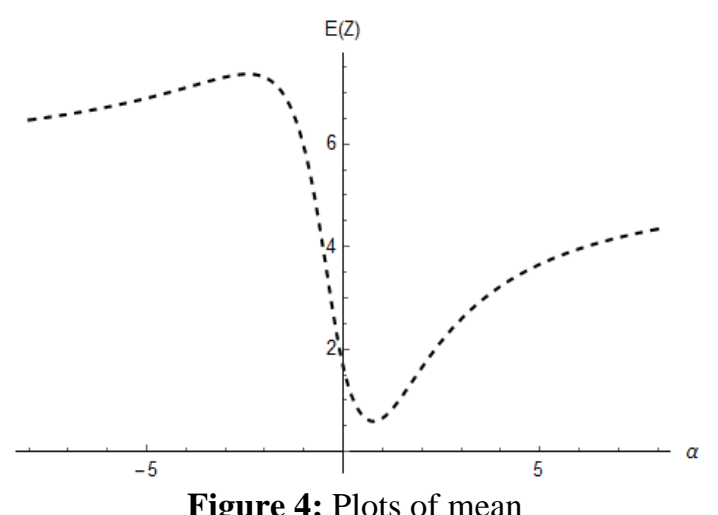

Figure 4: Plots of mean

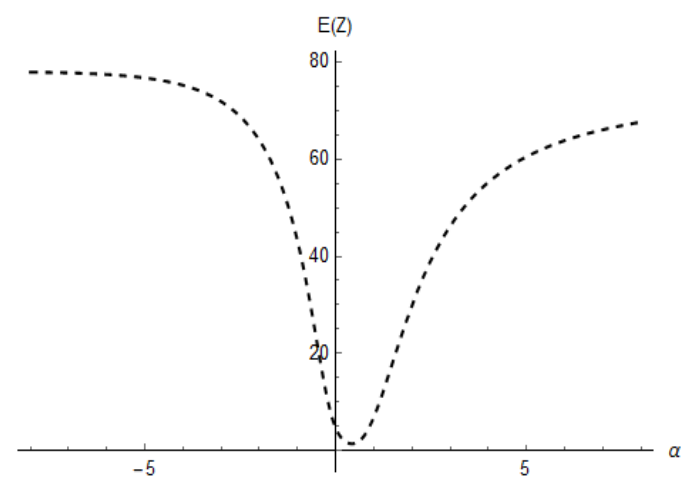

Figure 5: Plots of variance

Remark 3: The moments of $L B N(4)$ distribution can be derived easily by taking limit $\alpha \rightarrow \pm \infty$ in the moments of $\operatorname{LBASN}_{2}(\alpha)$ distribution so that $E(Z) \rightarrow 5.4957$ and $\operatorname{Var}(Z) \rightarrow 75.7007$.

\subsection{Skewness and Kurtosis}

The skewness and kurtosis of $\operatorname{LBASN}_{2}(\alpha)$ distribution are given by

$$
\begin{aligned}
& \beta_{1}=\frac{1}{C^{3}}\left[4 \left(e^{3}\left(C_{2}(\alpha)\right)^{2}\left[2+\alpha\left(-12+\alpha\left(40-72 \alpha+69 \alpha^{2}\right)\right)\right]+8[2+\alpha(-4+\alpha(8+\alpha(-8+5 \alpha)))]^{3}-\right.\right. \\
& \left.3 e C_{2}(\alpha)[2+\alpha(-4+\alpha(8+\alpha(-8+5 \alpha)))][4+\alpha(-16+\alpha(40+\alpha(-56+43 \alpha)))]^{2}\right]
\end{aligned}
$$
and

$$
\begin{aligned}
& \beta_{2}=\frac{1}{C^{2}}\left[-16 e^{3}\left(C_{2}(\alpha)\right)^{2}\left[2+\alpha\left(-12+\alpha\left(40-72 \alpha+69 \alpha^{2}\right)\right)\right][2+\alpha(-4+\alpha(8+\alpha(-8+5 \alpha)))]-\right. \\
& 48[2+\alpha(-4+\alpha(8+\alpha(-8+5 \alpha)))]^{4}+24 e C_{2}(\alpha)[2+\alpha(-4+\alpha(8+\alpha(-8+5 \alpha)))]^{2}[4+ \\
& \left.\alpha(-16+\alpha(40+\alpha(-56+43 \alpha))]+e^{6}\left(C_{2}(\alpha)\right)^{3}[4+\alpha(-32+\alpha(136+\alpha(-304+355 \alpha)))]\right] \\
& \text { where } C=[-4(2+\alpha(-4+\alpha(-8+5 \alpha)))]^{2}+e C_{2}(\alpha)[4+\alpha(-16+\alpha(40+\alpha(-56+43 \alpha)))] .
\end{aligned}
$$

The bounds for skewness and kurtosis can be derived by numerically optimizing $\beta_{1}$ and $\beta_{2}$ with respect to $\alpha$ as $15.9462 \leq \beta_{1} \leq 555.709$ and $46.8883 \leq \beta_{2} \leq 1543.16$. Also, to study their behavior we have plotted the skewness and kurtosis respectively in Figure (6) and Figure (7). These plots also verify these bounds.

Remark 4: If we take the limit $\alpha \rightarrow \pm \infty$ in the results of $L B A S N_{2}(\alpha)$ distribution, then we can derive the skewness and kurtosis of $L B N(4)$ distribution as $\beta_{1} \rightarrow 17.1334$ and $\beta_{2} \rightarrow 48.5346$. 


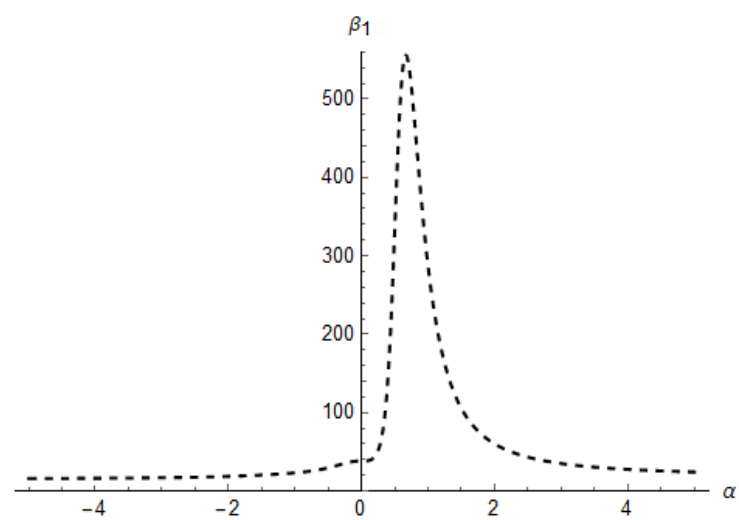

Figure 6: Plots of skewness

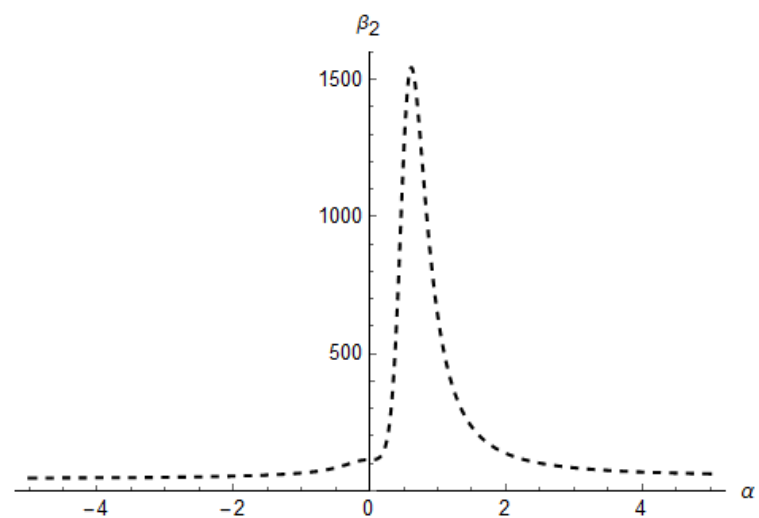

Figure 7: Plots of kurtosis

\section{Estimations and Illustrations with real datasets}

Here, we present the location $(\mu)$ and scale $(\sigma)$ extension of $L B A S N_{2}(\alpha)$ distribution which is given by

$$
f(z ; \alpha, \mu, \sigma)=\frac{\left[\left(1-\frac{\alpha(y-\mu)}{\sigma}\right)^{2}+1\right]^{2}}{z C_{2}(\alpha)} \phi\left(\frac{y-\mu}{\sigma}\right) ; z>0, \alpha \text { and } \mu \in R \text {, and } \sigma>0 \text {. }
$$

We denote it by $Y \sim \operatorname{LBASN}_{2}(\alpha, \mu, \sigma)$. In particular, for $\alpha \rightarrow \pm \infty, \operatorname{LBASN}_{2}(\alpha, \mu, \sigma) \rightarrow L B N$

\subsection{Method of moments}

The expressions for the moment estimators are relatively simple because the method needs to solve the following equations for $r=1,2,3$ to obtain moment estimates of $\mu, \sigma$, and $\alpha$.

$$
m_{r}=\frac{e^{\frac{r^{2}}{2}}\left[4-8 r \alpha+8 \alpha^{2}\left(1+r^{2}\right)-4 r \alpha^{3}\left(3+r^{2}\right)+\alpha^{4}\left(3+6 r^{2}+r^{4}\right)\right]}{4+8 \alpha^{2}+3 \alpha^{4}},
$$

where $m_{r}=\frac{1}{n} \sum_{i=1}^{n} z_{i}^{r}, r=1,2,3$. These equations can be simultaneously solved using numerical procedures available in the $\mathrm{R}$ software. This allows us to obtain the moment estimators $\left(\hat{\mu}_{m}, \hat{\sigma}_{m}, \hat{\alpha}_{m}\right)$ of $(\mu, \sigma, \alpha)$.

\subsection{Maximum likelihood estimation}

Let a random sample $y_{1}, y_{2}, \ldots, y_{n}$ of size $n$ be taken from $\operatorname{LBASN}_{2}(\alpha, \mu, \sigma)$ distribution of eqn.(6), then the loglikelihood function for $\theta=(\alpha, \mu, \sigma)$ is given by

$$
\begin{gathered}
l(\theta)=2 \sum_{i=1}^{n} \log \left[\left\{1-\alpha\left(\frac{y_{i}-\mu}{\sigma}\right)\right\}^{2}+1\right]-n \log \left(4+8 \alpha^{2}+3 \alpha^{4}\right)-n \log \sigma-\frac{n}{2} \log (2 \pi)-\sum_{i=1}^{n} y_{i}- \\
\frac{1}{2} \sum_{i=1}^{n}\left(\frac{y_{i}-\mu}{\sigma}\right)^{2} .
\end{gathered}
$$

On differentiating the eqn.(11) above partially with respect to the parameters $\alpha, \mu$, and $\sigma$ the following likelihood equations can be obtained as:

$$
\left.\begin{array}{l}
\frac{\partial l(\theta)}{\partial \mu}=-\sum_{i=1}^{n}-\frac{\left(y_{i}-\mu\right)}{\sigma^{2}}+2 \sum_{i=1}^{n} \frac{2 \alpha b_{i}}{\sigma\left(1+b_{i}{ }^{2}\right)} \\
\frac{\partial l(\theta)}{\partial \sigma}=-\frac{n}{\sigma}-\sum_{i=1}^{n}-\frac{\left(y_{i}-\mu\right)^{2}}{\sigma^{3}}+2 \sum_{i=1}^{n} \frac{2 \alpha\left(y_{i}-\mu\right) b_{i}}{\sigma^{2}\left(1+b_{i}{ }^{2}\right)} \\
\frac{\partial l(\theta)}{\partial \alpha}=-\frac{n\left(16 \alpha+12 \alpha^{3}\right)}{4+8 \alpha^{2}+3 \alpha^{4}}+2 \sum_{i=1}^{n}-\frac{2\left(y_{i}-\mu\right) b_{i}}{\sigma\left(1+b_{i}{ }^{2}\right)}
\end{array}\right\}
$$

where, $b_{i}=\left(1-\frac{\alpha\left(y_{i}-\mu\right)}{\sigma}\right)$.

Solving the above system of equations in eqn.(12) provides the maximum likelihood estimates for the parameters $\theta=$ $(\alpha, \mu, \sigma)$. The same can also be obtained by numerically maximizing eqn.(11) with respect to the parameters $\theta=$ $(\alpha, \mu, \sigma)$.

\subsection{Illustrations with real datasets}


Here we have considered two datasets. The first dataset is related to $\mathrm{N}$ latitude degrees in 69 samples from world lakes which appear in Column 5 of the Diversity data set in website:

http://users.stat.umn.edu/sandy/courses/8061/datasets/lakes.lsp. The second dataset consists of the velocities of 82 distant galaxies diverging from our own galaxy. The data set is available at

http://www.stats.bris.ac.uk/ peter/mixdata. We then compared the proposed distribution $\operatorname{LBASN}_{2}(\alpha, \mu, \sigma)$ with the $\log$-normal $\operatorname{LN}\left(\mu, \sigma^{2}\right)$ distribution, the $\log$-skew-normal $\operatorname{LSN}(\lambda, \mu, \sigma)$ distribution, and the log-alpha-skew-normal $\operatorname{LASN}(\alpha, \mu, \sigma)$ distribution of Venegas et al. (2016). The MLE of the parameters are obtained by using numerical optimization routine. AIC and BIC are used for model comparison.

Table 1: MLE's, log-likelihood, AIC and BIC for N latitude degrees in 69 samples from world lakes.

\begin{tabular}{|c|c|c|c|c|c|c|c|}
\hline $\begin{array}{l}\text { Parameters } \\
\text { Distributions }\end{array}$ & $\rightarrow$ & $\sigma$ & $\lambda$ & $\alpha$ & $\log L$ & AIC & $\mathrm{BIC}$ \\
\hline$N\left(\mu, \sigma^{2}\right)$ & 45.165 & 9.549 & -- & -- & -253.599 & 511.198 & 515.666 \\
\hline$L N\left(\mu, \sigma^{2}\right)$ & 3.791 & 0.189 & -- & -- & -244.612 & 493.223 & 497.692 \\
\hline $\operatorname{LSN}(\lambda, \mu, \sigma)$ & 3.599 & 0.269 & 2.343 & -- & -240.268 & 486.536 & 493.238 \\
\hline $\operatorname{LASN}(\alpha, \mu, \sigma)$ & 3.926 & 0.156 & -- & 1.780 & -232.298 & 470.596 & 477.298 \\
\hline $\operatorname{LBASN}_{2}(\alpha, \mu, \sigma)$ & 3.972 & 0.134 & -- & 1.743 & -229.053 & 464.106 & 470.809 \\
\hline
\end{tabular}

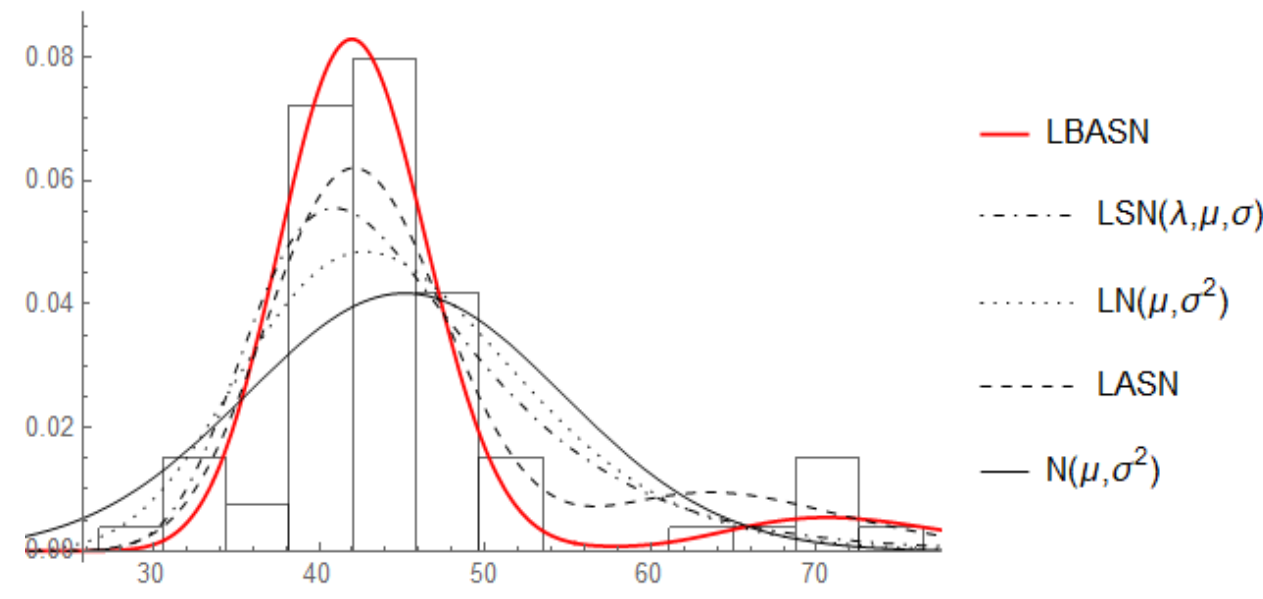

Figure 8: Plots of observed and expected densities for $\mathrm{N}$ latitude degrees in 69 samples from world lakes.

Table 2: MLE's, log-likelihood, AIC and BIC for the velocities of 82 distant galaxies diverging from our own galaxy.

\begin{tabular}{|c|l|c|l|l|l|l|c|}
\hline Parameters & $\rightarrow$ & & & & & \\
Distributions & \rfloor$^{\mu}$ & $\sigma$ & $\lambda$ & $\alpha$ & $\log L$ & AIC & BIC \\
\hline$L N\left(\mu, \sigma^{2}\right)$ & 3.007 & 0.258 & -- & -- & -251.937 & 507.874 & 512.687 \\
\hline$L S N(\lambda, \mu, \sigma)$ & 3.262 & 0.363 & -2.735 & -- & -242.505 & 491.011 & 498.231 \\
\hline$N\left(\mu, \sigma^{2}\right)$ & 20.832 & 4.540 & -- & -- & -240.420 & 484.833 & 489.646 \\
\hline$L A S N(\alpha, \mu, \sigma)$ & 2.806 & 0.214 & -- & -1.992 & -230.730 & 467.460 & 474.681 \\
\hline$L B A S N_{2}(\alpha, \mu, \sigma)$ & 2.739 & 0.185 & -- & -1.973 & $\mathbf{- 2 1 7 . 7 1 6}$ & $\mathbf{4 4 1 . 4 3 2}$ & $\mathbf{4 4 8 . 6 5 2}$ \\
\hline
\end{tabular}




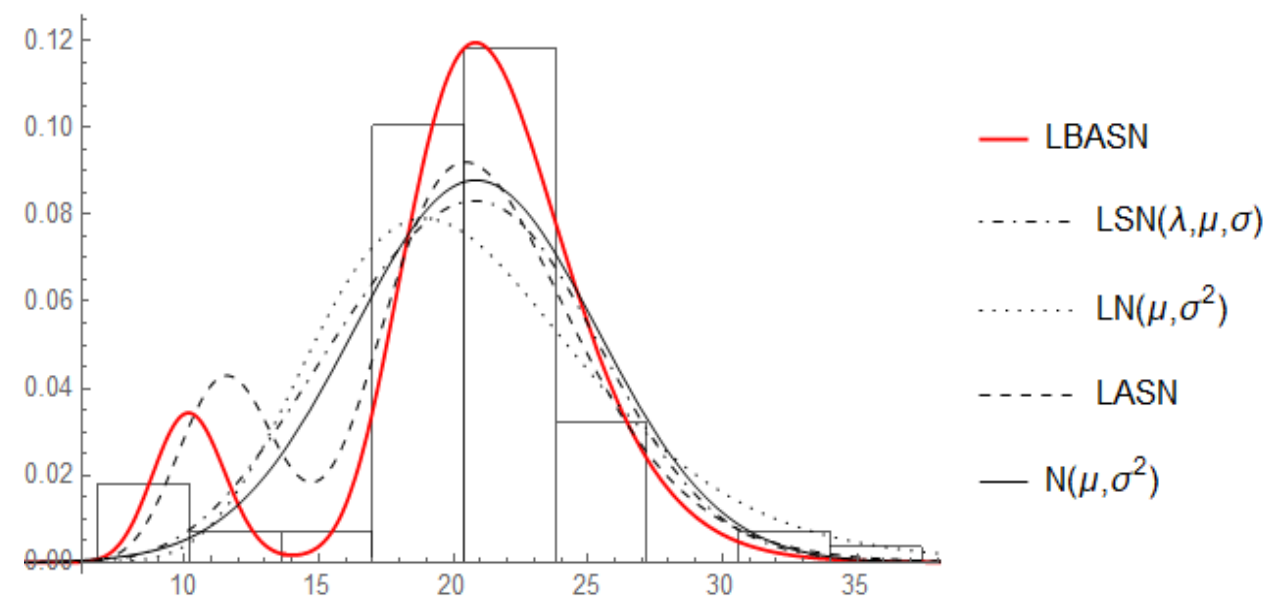

Figure 9: Plots of observed and expected densities for the velocities of 82 distant galaxies diverging from our own galaxy.

From Tables 1 and 2 , it is observed that the proposed log-Balakrishnan-alpha-skew-normal $\operatorname{LBASN}_{2}(\alpha, \mu, \sigma)$ distribution provides much better fit to the data sets under consideration in terms of the loglikelihood, AIC and BIC. Again, the plots of observed (in histogram) and expected densities (lines) presented in Figure 8 and Figure 9, also confirms our findings.

\subsection{Likelihood Ratio Test}

Further, since $L N\left(\mu, \sigma^{2}\right)$ and $\operatorname{LBASN}_{2}(\alpha, \mu, \sigma)$ are nested models, the likelihood ratio (LR) test is used to discriminate between them. The LR test is carried out to test the following hypothesis, $H_{0}: \alpha=0$, that is the sample is drawn from $\operatorname{LN}\left(\mu, \sigma^{2}\right)$ : against the alternative $H_{1}: \alpha \neq 0$, that is the sample is drawn from $\operatorname{LBASN}_{2}(\alpha, \mu, \sigma)$.

Table 3: The values of LR test statistic.

\begin{tabular}{|l|c|c|c|c|}
\hline & Dataset 1 & Dataset 2 & $\begin{array}{c}\text { Degrees of } \\
\text { Freedom }\end{array}$ & $\begin{array}{c}\text { Critical } \\
\text { values }\end{array}$ \\
\hline LR test statistic values & 31.117 & 68.442 & 1 & 6.635 \\
\hline
\end{tabular}

The values of LR test statistic for two datasets are respectively, 31.117 and 68.442 which exceed the critical value at $5 \%$ level of significance. Thus there is evidence in favor of the alternative hypothesis. Therefore, we may conclude that the sampled data come from $\operatorname{LBASN}_{2}(\alpha, \mu, \sigma)$ and not from $\operatorname{LN}\left(\mu, \sigma^{2}\right)$ in both cases.

\section{Concluding remark}

In this article the log-Balakrishnan-alpha-skew-normal distribution which has at most two modes is introduced and some of its basic properties are investigated. The numerical results of the modelling of two real life data sets considered here has shown that the proposed distribution $\operatorname{LBASN}_{2}(\alpha, \mu, \sigma)$ provides much better fit in comparison to the lognormal $L N\left(\mu, \sigma^{2}\right)$ distribution, the $\log$-skew-normal $\operatorname{LSN}(\lambda, \mu, \sigma)$ distribution and the log-alpha-skew-normal $\operatorname{LASN}(\alpha, \mu, \sigma)$ distribution. It is therefore expected that the proposed distribution will be useful for modelling different types of data.

\section{Acknowledgements}

The authors would like to acknowledge the editor(s) and anonymous referees for suggestions which helped to make the substantial improvements to the paper.

\section{References}

1. Ahrens, L. (1953). A fundamental law of geochemistry. Nature. 172(4390), 1148.

2. Ahrens, L. (1954a). The lognormal distribution of the elements.Geochimica et Cosmochimica acta 5, 49-73.

3. Ahrens, L. (1954b). The lognormal distribution of the elements. Geochimica et Cosmochimica acta 6, 121-131.

4. Arnold, B. C., and Beaver, R. J. (2002). Skewed multivariate models related to hidden truncation and/or selective reporting. Test, 11(1), 7-54. 
5. Azzalini, A. (1985). A class of distributions which includes the normal ones. Scandinavian Journal of Statistics,12: 171-178.

6. Azzalini, A, Dal Cappello T, and Kotz S. (2003). Log-skew-normal and log-skew-t distributions as models for family income data. Journal of Income Distribution, 11(3-4), 12-20.

7. Balakrishnan, N. (2002). Discussion on "Skew multivariate models related to hidden truncation and/or selective reporting" by B. C. Arnold and R. J. Beaver. Test,11:37-39

8. Elal-Olivero, D. (2010). Alpha-skew-normal distribution. Proyecciones (Antofagasta), 29(3), 224-240.

9. Hazarika, P. J., Shah, S., and Chakraborty, S. (2019). Balakrishnan Alpha Skew Normal Distribution: Properties and Applications. arXiv:1906.07424 [math.ST].

10. Huang, W. J., and Chen, Y. H. (2007). Generalized skew-Cauchy distribution. Statistics and Probability Letters, 77(11), 1137-1147.

11. Mateu-Figueras, G., Pawlosky-Glanh, and Barcelo-Vidal, C. (2004). The natural law in geochemistry: Lognormal or log skew-normal?, "32th International Geological Congress", International Union of Soil Sciences, Florence, Italy, 1849-1858.

12. Venegas, O., Bolfarine, H., Gallardo, D. I., Vergara-Fernández, A., and Gómez, H. W. (2016). A Note on the Log-Alpha-Skew-Normal Model with Geochemical Applications. Appl. Math, 10(5), 1697-1703.

13. Vistelius, A. B. (1960). The skew frequency distributions and the fundamental law of the geochemical processes. The Journalof Geology, 68 (1), 1-22. 